Investigaciones geográficas, $n .^{\circ} 45$ (2008) pp. 121-140 ISSN: 0213-4691
Instituto Universitario de Geografía Universidad de Alicante

\title{
ALCANCE Y LÍMITES DE LA DESINDUSTRIALIZACIÓN METROPOLITANA: EL CASO DE ZARAGOZA (ESPAÑA)
}

\author{
A.I. Escalona Orcao y E. Climent López \\ Departamento de Geografía y Ordenación del Territorio \\ Universidad de Zaragoza
}

\section{Introducción}

Un rasgo característico de los mapas industriales contemporáneos es la vigencia de las ciudades y sus entornos como localizaciones predilectas para la mayor parte de las actividades industriales. La industria ya no es el principal motor del crecimiento metropolitano, pero las metrópolis no se desindustrializan (Holz y Houssel, 2003: 238). Por el contrario: «Virtualmente toda la actividad industrial se localiza en los lugares urbanos y sus regiones locales asociadas» (Dicken, 2004: 75). Se ha confirmado, además, que el patrón de localización industrial interurbano es altamente estable y que el descenso de los costes de transporte y telecomunicación no ha ido acompañado de una desconcentración significativa de las actividades industriales, sino de un refuerzo de la localización industrial en las ciudades (Storper y Venables, 2002).

Según una hipótesis sugestiva, las pautas de localización industrial en un sistema urbano se relacionan con los atributos típicos y diferenciados de los distintos tamaños de ciudades. Se asume que las grandes ciudades son ventajosas para empresas que buscan economías de aglomeración, mientras que las de tipo medio y pequeño son más atractivas para empresas que buscan bajos costes laborales y de suelo (Polèse y Shearmour, 2004). Pero ¿qué ocurre cuando una gran ciudad ofrece a la vez economías de aglomeración y bajos costes laborales y de suelo? Tal es la pregunta que se plantea en este artículo sobre la ciudad de Zaragoza y su entorno metropolitano, que constituye el espacio más relevante de todo el eje industrial del Valle del Ebro. 
Una serie de circunstancias peculiares hacen a Zaragoza diferente de otras grandes ciudades y metrópolis españolas y europeas (Nel-lo, 1997). Destaca en primer lugar la marcada macrocefalia de la red urbana, ya que no hay en todo el sector central del eje del Ebro ninguna ciudad que pueda competir con Zaragoza (664101 habitantes); las ciudades de Pamplona y Logroño (al noroeste) y Lérida (al este) están demasiado distantes y tienen una población sensiblemente menor. Por otra parte, debido a la gran extensión de su término municipal $-1060 \mathrm{~km}^{2}$ - Zaragoza cuenta con abundante suelo bien equipado para uso industrial a precios muy competitivos. Por último, hay un claro diferencial entre los salarios de Zaragoza y Aragón, por un lado, y los de las regiones más industriales de España (País Vasco, Cataluña y Madrid), de las que dista menos de 300 kilómetros (Alonso et al., 2006).

El artículo presenta los efectos de tales factores en la dinámica industrial metropolitana mediante un análisis del movimiento industrial registrado en Zaragoza y su entorno entre 1992 y 2005. En él se pone de relieve que la ciudad central continúa captando una proporción sustancial del crecimiento de la industria en el sector central del eje del Ebro. Ello se relaciona estrechamente con un segundo hecho llamativo, como es que el alcance espacial de la desconcentración industrial es muy limitado, superando en pocas ocasiones la isocrona de los 35 minutos. El análisis realizado permite concluir que en casos como el de Zaragoza, la dinámica industrial metropolitana no se explica como resultado de un dilema entre los factores de localización antes citados (economías de aglomeración y costes de producción), sino por la suma de los mismos.

\section{Consideraciones teóricas e implicaciones analíticas}

El estudio de la dinámica industrial metropolitana y el esclarecimiento de por qué las ciudades mantienen su importancia en la localización de la industria ha suscitado numerosos trabajos con diversos enfoques de la cuestión. El ya citado de Polèse y Shearmour (2004) y otros del mismo equipo (Polèse y Champagne, 1999; Polèse et al., 2005) se centran en observar la influencia de dos factores destacados por su potencial explicativo: las economías de aglomeración y los costes de interacción espacial. En relación con el primero sostienen como hipótesis que «la decisión de localizarse (dentro, cerca o lejos de una gran metrópoli) dependerá en gran parte de la importancia relativa para la empresa de las economías de aglomeración y los costes laborales y de suelo» (Polèse y Shearmour, 2004: 435). En relación con el segundo postulan que la desconcentración hacia ciudades más pequeñas depende de la influen- 
cia de la distancia a las metrópolis en los costes de interacción espacial de las compañías.

La comprobación empírica de tales hipótesis en estudios sobre Canadá y México permite concluir -en la línea de las investigaciones de Henderson (1994)- que las economías de aglomeración mantienen su importancia como factor de localización en las fabricaciones de alto valor añadido e intensivas en conocimiento, que obtienen los más altos cocientes de localización en las grandes ciudades, donde tales economías se manifiestan de forma más efectiva. En cambio las industrias de medio y bajo valor añadido, cuyas empresas son más sensibles al coste salarial y necesitan más espacio son impelidas a localizarse en ciudades más pequeñas (Polèse y Shearmour, 2004). No obstante, en ciudades del mismo tamaño los cocientes de localización más altos para toda clase de industrias se obtienen en las que se encuentran dentro del alcance de una metrópoli, por el deseo de las compañías de minimizar los costes de interacción espacial en los que incurrirían si se alejan. Queda claro que el alcance de la desconcentración industrial no sobrepasa el umbral de distancia de 100 a $150 \mathrm{~km}$ (o una hora) desde la ciudad principal, dependiendo de las condiciones de las vías de transporte. Se confirma así que la proximidad a una gran ciudad también cuenta y que la distancia disuade a las empresas a la hora de localizarse y limita los procesos de desconcentración industrial (Polèse y Champagne, 2002; Polèse y Shearmour, 2004).

En una investigación sobre España (Polèse et al., 2005) se obtienen resultados semejantes. Los valores del cociente de localización para actividades manufactureras descienden en las grandes ciudades (las de más de 500000 habitantes) pero aumentan en las demás categorías de forma inversa a su tamaño: son más altos en las ciudades más pequeñas. La proximidad a una gran ciudad se revela de nuevo como factor de localización más importante que la dimensión urbana, ya que los cocientes altos corresponden siempre a ciudades situadas a menos de una hora de distancia de una metrópoli o gran ciudad. Quedan así refrendados los procesos de desconcentración industrial, dentro del umbral señalado, como tendencia dominante en todas las aglomeraciones metropolitanas españolas, algo resaltado también en otros estudios (Méndez et al., 2005)

Conviene recordar que los resultados dependen de la metodología y ésta, a su vez, de la escala del estudio. En todas las investigaciones citadas la escala es nacional y requiere la agregación de todas las ciudades en un número reducido de categorías. Los cocientes de localización que indican la intensidad de la industrialización media en cada categoría se obtienen generalmente a par- 
tir de clases muy heterogéneas ${ }^{1}$. Por otra parte, debido a las determinaciones analíticas relativas a la escala y al uso de datos agregados para describir las grandes aglomeraciones, resulta difícil apreciar la existencia de más procesos industriales que los de desconcentración, tales como nuevas implantaciones industriales de carácter centrípeto que compensen los movimientos de tipo centrífugo o los traslados intrametropolitanos. Ya en 1997 Graham y Spence afirmaban respecto del área metropolitana de Londres que «ciertas empresas industriales aún quieren estar en Londres ... y que las dinámicas industriales metropolitanas son complejas y no pueden reducirse únicamente a un proceso de declive y descentralización progresiva» (Graham y Spence, 1997: 482-483).

De lo hasta ahora dicho pueden deducirse algunas implicaciones analíticas relevantes para este estudio sobre la dinámica industrial metropolitana en Zaragoza. La primera es que debe prestarse atención a las características específicas del sistema urbano regional, ya que obviamente los procesos de desconcentración se ven facilitados cuando la red urbana tiene ciudades suficientes en todos los niveles jerárquicos (y a la inversa). En segundo lugar deben analizarse las ventajas competitivas del área, no sólo las atribuidas a ciudades grandes -la oferta de economías de aglomeración y la buena interacción espacial- sino también otras susceptibles de atraer determinados tipos de empresas, incluidas las sensibles al coste salarial y las que necesitan más espacio. Y ello se relaciona con la tercera implicación: que la escala del estudio sea lo suficientemente grande como para que se manifiesten las variaciones locales de la dinámica metropolitana; para ello se requieren datos que diferencien adecuadamente y a esa escala los tipos básicos de movimientos o de operaciones industriales, es decir, las nuevas implantaciones y los traslados. Tales determinaciones metodológicas se aplican en el análisis que sigue.

\section{El espacio industrial metropolitano de Zaragoza. Presentación y ventajas competitivas}

Zaragoza se encuentra en el centro del cuadrante nororiental de España, equidistante de las cuatro principales aglomeraciones urbano-industriales del país (figura 1). A esta situación, considerada como privilegiada en el contexto español, se ha atribuido que la ciudad y su entorno haya experimentado desde

1. A modo de ejemplo, en la segunda categoría del estudio citado sobre España (Polèse et al., 2005), la de Zaragoza es la aglomeración más pequeña, con sólo dos municipios y 664000 habitantes, siendo la de Valencia la mayor con 22 veces más municipios (44) y 2,2 veces más población (1460000). Otras aglomeraciones que forman parte de esta categoría son las de Sevilla (con 25 municipios y 1211041 habitantes), Bilbao (35 y 1679800), Asturias (18 y 556200) y Málaga (siete y 1077 700) 
Figura 1: Red urbana en la región del valle del Ebro

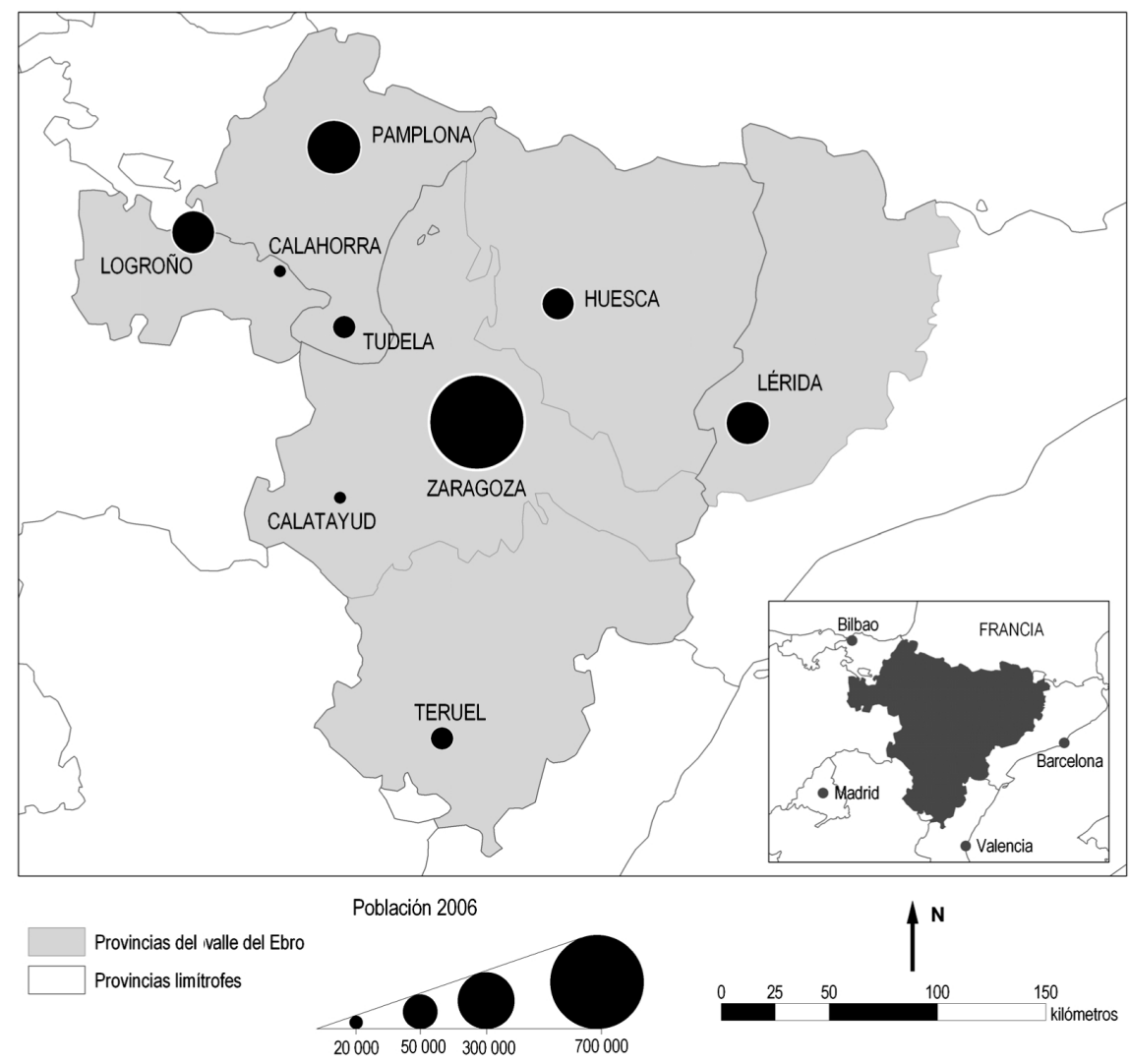

fecha temprana un importante desarrollo industrial. Probablemente el hito más destacado sea la implantación en 1981 de una factoría de General Motors a $28 \mathrm{~km}$ de la ciudad, la cual está en el origen de un clúster de industrias del automóvil que ha ido aumentando su importancia desde entonces. En el año 2005 la provincia de Zaragoza era una de las más industrializadas de España, siendo la sexta por el valor de la producción industrial y la séptima por número de trabajadores (Alcaide y Alcaide, 2007)

\subsection{Ventajas competitivas vinculadas a las economías de aglomeración}

La red urbana de las regiones del valle del Ebro adolece de una fuerte macrocefalia (tabla 1). Las ciudades que podrían competir con Zaragoza en cuanto a la localización de industrias y participar en un proceso de desconcentración 
Tabla 1: Jerarquía urbana en las regiones del valle del Ebro (ciudades con más de 20000 habitantes)

\begin{tabular}{|c|l|c|c|}
\hline Rango & Ciudad & Población (2006) & $\begin{array}{c}\text { Distancia a } \\
\text { Zaragoza (km) }\end{array}$ \\
\hline 1 & Zaragoza * & 664101 & 0 \\
\hline 2 & Pamplona ** & 260050 & 179 \\
\hline 3 & Logroño & 147036 & 177 \\
\hline 4 & Lérida & 125677 & 151 \\
\hline 5 & Huesca & 49312 & 72 \\
\hline 6 & Teruel & 33673 & 184 \\
\hline 7 & Tudela & 32802 & 86 \\
\hline 8 & Calahorra & 23708 & 127 \\
\hline 9 & Calatayud & 20001 & 87 \\
\hline
\end{tabular}

* Se ha incluido el municipio de Utebo, contiguo al municipio central.

** Se han incluido los municipios de Barañain, Burlada, Villaba y Zizur Mayor, contiguos al municipio central.

Fuente: Instituto Nacional de Estadística <http://www.ine.es>.

de tipo jerárquico están demasiado alejadas o son demasiado pequeñas o ambas cosas.

El tamaño de Zaragoza y su fuerte primacía dentro de la red urbana convierten a la ciudad en fuente sin igual de economías de aglomeración, tales como el acceso a un amplio mercado de trabajo y a un amplio rango de servicios a las empresas. Estas circunstancias han contribuido al desarrollo de la industria metropolitana en el pasado (Villaverde, 2001; López et al, 2002; Méndez y Sánchez, 2003) y se han visto reforzadas en la actualidad. Por ejemplo, la construcción y entrada en servicio de los nuevos cinturones de circunvalación de la ciudad, del tren de alta velocidad Madrid-Zaragoza-Barcelona, o la revitalización del aeropuerto de Zaragoza han contribuido a reducir más todavía los costes de transporte y a maximizar las ventajas tradicionales de interacción espacial. A ello se añade el desarrollo de equipamientos logísticos sofisticados, como la plataforma logística de Zaragoza PLA-ZA, que convierten a la ciudad en un hub, o centro de distribución nacional e internacional de importantes grupos empresariales, entre los que cabe citar Inditex -propietario de la conocida marca Zara- e Imaginarium, líderes mundiales en la distribución de prendas de vestir y de juguetes, respectivamente, y DHL, multinacional de mensajería, entre otras (Alonso et al., 2006)

Los costes de transacción con clientes y proveedores también han disminuido y favorecido la implantación empresarial. El acceso a mercados, en 
particular, influye en la localización de empresas de tamaño medio (de más de 20 trabajadores) que se han trasladado recientemente a Zaragoza y su entorno próximo (Escalona et al., 2006). Y la proximidad a proveedores es particularmente apreciada por las empresas pertenecientes a los sectores de productos metálicos y maquinaria localizadas tanto en Zaragoza como en municipios de su entorno inmediato. Otros dos hechos recientes confirman la importancia de este factor de localización para Zaragoza y su entorno. El primero es que la red de proveedores generada en torno a la factoría de General Motors resultó decisiva para que la factoría aragonesa se impusiera a una polaca de la misma empresa en el litigio por la fabricación de un nuevo modelo de automóvil que habrá de garantizar la continuidad de la factoría a corto y medio plazo. El segundo es la existencia de empresas trasladadas a Zaragoza desde regiones limítrofes ya que su excelente accesibilidad les permite mantener su red de proveedores habitual.

Por otra parte Zaragoza concentra el $81 \%$ de los empleos y el 70,6\% de las empresas de servicios avanzados de toda la región (Pardos et al., 2004). Las evidencias empíricas indican que la importancia, como factor de localización, del fácil acceso a proveedores de servicios a las empresas no es grande. Ello puede estar relacionado con el pequeño tamaño medio de las empresas en la zona, ya que el $74 \%$ de las mismas tiene menos de 10 trabajadores y el 54\% menos de seis. Por el mismo motivo, aunque la aglomeración ofrece acceso al principal mercado de trabajo de la región, la disponibilidad de mano de obra no es un factor particularmente relevante en las decisiones de localización de la mayoría de las empresas, con la excepción de las industrias metálicas y del automóvil, donde la ventaja de Zaragoza como proveedor de mano de obra cualificada es altamente apreciada (Escalona et al., 2006).

\subsection{Otras ventajas competitivas: costes salariales y precio del suelo}

Junto con las economías de urbanización Zaragoza ofrece las ventajas de localización que las empresas obtienen al localizarse en ciudades más pequeñas en mayor medida que al hacerlo en otras más grandes. La primera es el coste de la mano de obra, sensiblemente inferior al de regiones vecinas de mayor peso industrial, como Cataluña y País Vasco. Según diversas estimaciones los costes por trabajador son 1,23 veces más caros en el País Vasco y 1,06 en Cataluña. Por otra parte el precio del suelo es un 30\% más económico en Aragón que en las comunidades citadas y el de las naves industriales puede ser hasta 
un $70 \%$ menor $^{2}$. Ello se relaciona con la extensión del término municipal de la capital $\left(1052 \mathrm{~km}^{2}\right)$, uno de los mayores de España, y con la proliferación reciente de espacios adecuados para paliar lo que se percibía como un déficit crónico de equipamientos industriales de calidad ${ }^{3}$.

También los municipios del entorno apuestan por ampliar su oferta de suelo industrial acondicionado, promoviendo polígonos de carácter público, apoyando a los de iniciativa privada y facilitando la instalación de industrias en suelo no ordenado cuando aprecian que se trata de iniciativas de interés social. Investigaciones recientes confirman una alta influencia del factor precio del suelo así como de la dotación de los polígonos industriales en las decisiones de localización tomadas por las empresas (Escalona et al., 2007). Los municipios adyacentes a Zaragoza que ofrecen suelo a bajo coste son muy competitivos a la hora de atraer empresas que desean trasladarse. No entran en este perfil únicamente empresas tradicionales en busca de suelo barato (Méndez, 2002), sino también empresas avanzadas que realizan fuertes inversiones en suelo para hacer frente a previsiones de expansión a corto y medio plazo $^{4}$. Es interesante ver que en ambas operaciones han recibido apoyo del gobierno regional y de los respectivos ayuntamientos. En cuanto a la dotación del polígono, es un factor valorado por empresas que pertenecen a sectores de los considerados de demanda débil y que se han establecido recientemente en municipios del entorno de Zaragoza (Escalona, et al., 2007).

\subsection{La influencia de los promotores privados y de la política industrial regional en la dinámica metropolitana}

El hecho de que un importante número de empresas destacadas a escala nacional en la promoción de inmuebles de uso industrial hayan abierto recientemente delegación en Zaragoza está claramente relacionado con el incremento de las posibilidades industriales. La mayor parte de la inversión que realizan consiste todavía, a diferencia de lo observado en las grandes ciudades españolas, en la compra de suelo industrial (y de naves de diversos tipos) en los

2. Datos aportados por el director del Instituto Aragonés de Fomento en su comparecencia ante las Cortes de Aragón reseñada en El Periódico de Aragón, de 12 de marzo de 2006.

3. Es interesante mencionar que el Plan General de Ordenación Urbana de Zaragoza, aprobado en 2002, establece aumentar en un $77 \%$ el suelo ocupado por la industria en el término municipal, así como acometer diversas mejoras en equipamientos, infraestructuras y accesos (Alonso et al., 2006).

4. Sirvan de ejemplo los casos de algunas importantes empresas metalúrgicas que se han trasladado a los municipios de Zuera (eje norte) y Épila (eje suroeste) tras adquirir, respectivamente, 50000 y 10000 metros cuadrados de suelo. 
principales polígonos del área, para su posterior venta o alquiler a las empresas. Los promotores se convierten aquí, más tarde que en otras áreas urbanas del país, en agentes inductores de las decisiones de localización industrial, anticipándose a la demanda y orientando las elecciones de los empresarios hacia los emplazamientos mejor equipados. Se verá después que ello ha contribuido a mantener la importancia industrial de la ciudad de Zaragoza, ya que los polígonos nuevos del término municipal, así como los de su entorno más próximo, siguen captando una alta proporción de las inversiones totales.

No menos importante es la gestión pública para atraer inversiones, sobre todo de empresas foráneas 5 . Elemento fundamental en la atracción de las empresas es la subvención que reciben (20\% sobre el total de la inversión en activos fijos), aunque también son relevantes algunos de los factores antes citados, como los menores costes laborales y de suelo, la cultura empresarial o la presencia de proveedores. Entre 2006 y 2008 se ha conseguido atraer hasta Aragón a una treintena de empresas españolas, de las que el $76 \%$ son catalanas, y una veintena de empresas extranjeras, de las que el $25 \%$ son italianas. El 37,5\% de las empresas beneficiarias de los incentivos se sitúa en los corredores de expansión de Zaragoza. Algunas estaban implantadas ya en España con anterioridad, de modo que su llegada a la zona metropolitana de Zaragoza suele coincidir con procesos de ampliación o de reorganización de su mapa productivo: cierre de la factoría anterior y traslado de la producción a plantas zaragozanas, nuevas o ya existentes ${ }^{6}$.

\section{Pautas de localización industrial metropolitana. Análisis del movimiento industrial}

Para comprobar los efectos de las ventajas competitivas citadas sobre la dinámica industrial metropolitana y el alcance de los procesos de desconcentración industrial, se ha llevado a cabo un análisis de las implantaciones industriales registradas entre 1992 y 2005 en un área formada por Zaragoza y 29

5. Para contrarrestar el peso de las actividades ligadas al sector del automóvil en la región, mejorar la competitividad de sectores motrices, corregir los riesgos de deslocalización y poner en valor el territorio, la Administración aragonesa ha puesto en marcha el programa DIVERSIA, encomendado al Instituto Aragonés de Fomento (IAF, 2006). A él se añaden las iniciativas del lobby Aragonex, también impulsado por el gobierno regional y responsable de la captación de empresas extranjeras.

6. Legger y Platt, multinacional norteamericana dedicada a la fabricación de colchones, es un ejemplo: cierra su planta de Zamudio (Vizcaya) y traslada la producción a la planta que ya tenía en Cadrete, al sur de Zaragoza. Otro caso es el de Jacquet Ibérica, cuya planta de producción y distribución de derivados del metal en Épila (corredor suroeste), viene precedida del cierre del centro de distribución que la empresa tenía en Barcelona. 
Figura 2: Localización de las empresas de nueva creación y de las empresas trasladadas en el entorno metropolitano de Zaragoza

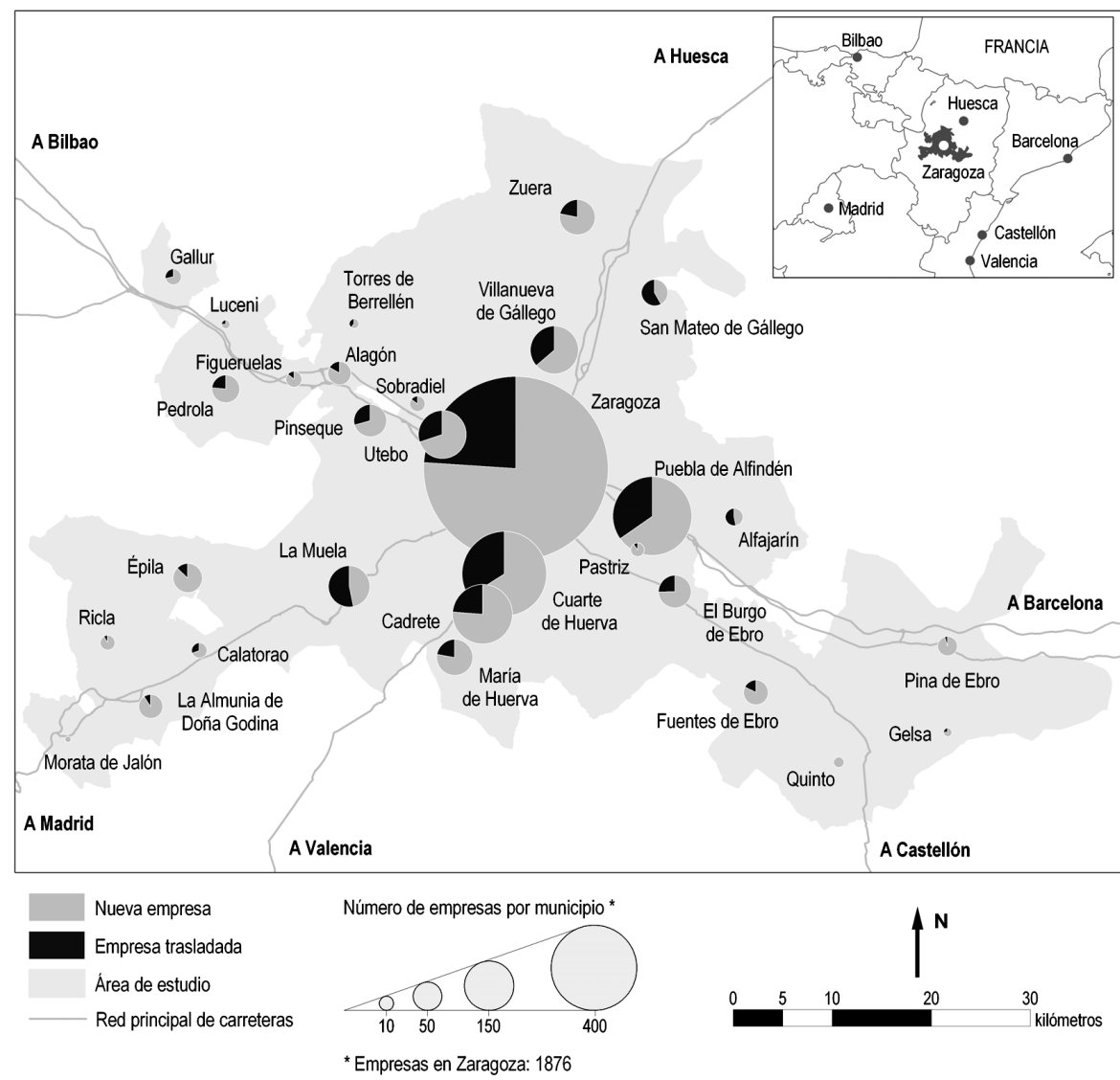

municipios de su entorno, situados en los seis ejes de expansión intrametropolitanos (figura 2): hacia Huesca en dirección norte, Barcelona al nordeste, Castellón al sureste, Valencia al sur, Madrid al suroeste y Bilbao al noroeste. Los municipios seleccionados son aquellos en que se ha registrado al menos una empresa con 20 empleados y que tienen al menos 70 empleos industriales. La fuente de información utilizada ha sido el Registro de Establecimientos Industriales, que recoge un total de 3998 empresas establecidas en la zona en el período de estudio, lo cual representa una media de 268 empresas por año. Los empleos creados en el área fueron 32009, lo cual da una media de 8,5 empleos por empresa. 


\subsection{La dinámica metropolitana según las características de las empresas}

$y$ de las áreas

Tal como se explicó en el apartado teórico, las grandes ciudades generan procesos de desconcentración industrial porque muchas empresas son impulsadas a localizarse en ciudades más pequeñas. La hipótesis que se quiere demostrar en este capítulo va más en la línea del trabajo de Graham and Spencer (1987): la dinámica metropolitana no consiste sólo en movimientos centrífugos, sino que se trata de un fenómeno más complejo. El punto de partida del análisis es la tabla 2, que ilustra dos contrastes muy interesantes: entre empresas (de nueva creación y trasladadas) y entre áreas (la ciudad central y los ejes de expansión).

Es importante tener en cuenta que la ciudad de Zaragoza alberga al 50\% de las empresas industriales que se han establecido en el área desde 1992, las cuales aportan el $47 \%$ del empleo creado, cifras claramente superiores a las que cabría esperar en las ciudades de tamaño similar de las economías avanzadas. Más aún, el 76\% de las empresas registradas en la ciudad son nuevas, lo cual puede interpretarse como una evidencia de la existencia de movimientos centrípetos bastante intensos. En términos absolutos, la atracción del centro metropolitano para empresas de nueva creación es ligeramente mayor que la de los ejes de expansión industrial. Las empresas de nueva creación no registradas en el centro se localizan en sólo siete de los 29 municipios que componen dichos ejes.

La cuarta parte de las industrias registradas en el área son fruto de traslados y su tamaño es superior a la media, correspondiéndoles el 44\% del empleo

Tabla 2: Empresas y empleos industriales en el entorno metropolitano de Zaragoza

\begin{tabular}{|c|c|c|c|c|c|c|c|c|c|c|c|c|}
\hline \multirow{3}{*}{$\begin{array}{l}\text { Ámbito } \\
\text { espacial }\end{array}$} & \multicolumn{6}{|c|}{ Empresas } & \multicolumn{6}{|c|}{ Empleos } \\
\hline & \multicolumn{2}{|c|}{ Nuevas } & \multicolumn{2}{|c|}{ Traslados } & \multicolumn{2}{|c|}{ Total } & \multicolumn{2}{|c|}{ Nuevas } & \multicolumn{2}{|c|}{ Traslados } & \multicolumn{2}{|c|}{ Total } \\
\hline & Total & $\%$ & Total & $\%$ & Total & $\%$ & Total & $\%$ & Total & $\%$ & Total & $\%$ \\
\hline $\begin{array}{l}\text { Ciudad de } \\
\text { Zaragoza }\end{array}$ & 1426 & 51,9 & 450 & 43,8 & 1876 & 49,7 & 7710 & 42,9 & 7411 & 52,8 & 15121 & 47,2 \\
\hline $\begin{array}{l}\text { Corredores de } \\
\text { expansión }\end{array}$ & 1319 & 48,0 & 577 & 56,2 & 1896 & 50,3 & 10272 & 57,1 & 6616 & 47,2 & 16888 & 52,8 \\
\hline $\begin{array}{l}\text { Total entorno } \\
\text { metropolitano }\end{array}$ & 2745 & 100 & 1027 & 100 & 3772 & 100 & 17982 & 100,0 & 14027 & 100 & 32009 & 100,0 \\
\hline
\end{tabular}

Fuente: Registro de Establecimientos Industriales (1992-2005).

Investigaciones geográficas, n. ${ }^{\circ} 45$ (2008) 
total creado. Aunque el traslado de industrias es un factor de la desconcentración metropolitana, en este estudio el $44 \%$ de las empresas trasladadas aparece registrado en la ciudad de Zaragoza, centro metropolitano (tabla 2). Las empresas estuvieron originalmente localizadas en las áreas industriales más antiguas de la ciudad, con instalaciones obsoletas, inadecuadas para los métodos recientes de producción y gestión, y se desplazaron a los nuevos polígonos industriales del municipio, donde pudieron conservar su fuerza de trabajo, proveedores y clientes originales, ampliar sus instalaciones y adaptarlas a sus necesidades actuales y futuras. En particular hay tres nuevos polígonos situados al sur de la ciudad, pero dentro de su término municipal: la Plataforma Logística de Zaragoza (PLAZA), con 1300 hectáreas y financiada al 70\% con dinero público; el Parque Tecnológico del Reciclado, una iniciativa privada con 850 hectáreas, y el polígono Empresarium, también de iniciativa privada, con 750 hectáreas. Estas promociones de suelo han sido las responsables de una enorme expansión industrial en el interior del centro metropolitano desde 2002 (Alonso et al., 2006).

La proporción de empresas venidas de fuera es todavía muy baja en estos polígonos. A corto plazo es muy probable que continúe el traslado a ellos de empresas procedentes de las áreas industriales antiguas de la ciudad, estimulado por la mediación del Ayuntamiento y la firma de acuerdos relativos a los futuros usos del suelo ${ }^{7}$. Aunque éstos son típicos casos de traslado a corta distancia desde el emplazamiento original y no constituyen realmente una deslocalización industrial porque las empresas no abandonan el término de Zaragoza.

En los municipios que rodean la ciudad la proporción de empresas trasladadas aumenta ligeramente, siendo de un tamaño relativamente mayor, porque cuentan con el $40 \%$ del empleo generado, si bien el tamaño medio es todavía bastante pequeño (11,6 trabajadores por empresa). Los resultados de una encuesta muestran que la mayoría de las empresas trasladadas proceden también de Zaragoza, confirmando la existencia de fuerzas centrífugas de más amplio radio que en el caso anterior (Escalona et al., 2006). No obstante, el alcance del proceso es limitado: el 85\% de los traslados se registran en sólo siete municipios adyacentes o muy cercanos a Zaragoza (menos de $20 \mathrm{~km}$ ). Todos ellos han mejorado recientemente su oferta de suelo industrial, como, por ejemplo, La Muela, en el corredor suroeste, Villanueva de Gállego y San Mateo de Gállego, ambos en el corredor norte.

7. Ejemplos de esta tendencia serían una fábrica local de cervezas, con más de $180 \mathrm{em}-$ pleados o una multinacional de la fabricación de ascensores con una plantilla de unas 400 personas. 
La encuesta también ha revelado la existencia de movimientos intra-corredores, tales como los de Cuarte de Huerva a Cadrete (corredor sur) o los de Utebo a Pinseque (corredor noroeste). Esto sugiere que ya hay escasez de suelo en los municipios más próximos a Zaragoza de los corredores sur y noroeste. Las empresas que necesitan ampliar sus fábricas están abandonando los enclaves industriales más deteriorados y ocupando el suelo libre y barato de los nuevos polígonos industriales de sus municipios vecinos; este proceso es similar al movimiento desde los barrios centrales de la ciudad hacia la periferia. La continuidad de este proceso estará determinada por las diferencias entre los municipios respecto a la existencia de suelo y a su precio.

El análisis deja claro que la ciudad de Zaragoza es todavía un centro muy atractivo, tanto para la localización de nuevas empresas como para el traslado de otras. Esto confirma la hipótesis inicial sobre la complejidad de los movimientos industriales en los centros metropolitanos: como se ha visto, no coinciden plenamente con lo establecido por la teoría de la desconcentración metropolitana.

\subsection{Especialización sectorial y preferencias de localización}

La tabla 3 muestra que las empresas que se dedican a fabricaciones de alto valor añadido son una proporción muy pequeña $(3,7 \%)$ de las registradas en el espacio y período estudiados. Es ésta la única categoría en la que se aprecia la tendencia internacional recogida por la teoría, dada su clara concentración en el centro metropolitano. Los cuatro tipos de industria incluidos en esta categoría -productos farmacéuticos, máquinas de oficina y equipos informáticos, componentes electrónicos y equipos e instrumentos medicoquirúrgicos y de precisión- incorporan un alto componente de conocimiento

Tabla 3: Localización de los nuevos establecimientos industriales según valor añadido

\begin{tabular}{|c|c|c|c|c|c|c|}
\hline \multirow[b]{2}{*}{ VALOR AÑADIDO } & \multicolumn{2}{|c|}{$\begin{array}{c}\text { Centro } \\
\text { metropolitano } \\
\text { (Zaragoza) }\end{array}$} & \multicolumn{2}{|c|}{$\begin{array}{l}\text { Corredores de } \\
\text { expansión }\end{array}$} & \multicolumn{2}{|c|}{ Total zona de estudio } \\
\hline & Total & \% Rama & Total & \% Rama & Total & $\%$ Industria \\
\hline Bajo & 1180 & 51,85 & 1096 & 48,15 & 2276 & 60,34 \\
\hline Medio & 593 & 43,64 & 766 & 56,36 & 1359 & 36,03 \\
\hline Alto & 103 & 75,18 & 34 & 24,82 & 137 & 3,63 \\
\hline Total industria & 1876 & 49,73 & 1896 & 50,27 & 3772 & 100,00 \\
\hline
\end{tabular}

Fuente: Registro de Establecimientos Industriales (1992-2005). 
y requieren, entre otros factores, la mano de obra altamente cualificada, los servicios empresariales avanzados y las excelentes comunicaciones que la ciudad de Zaragoza ofrece. No obstante también hay algunas empresas de este tipo en la periferia metropolitana, a una distancia que les permite beneficiarse plenamente de las ventajas citadas.

La mayor parte de las empresas $(60,34 \%)$ se dedican a fabricaciones de bajo valor añadido y se localizan no sólo en la periferia metropolitana-como prevé la teoría- sino también en el centro. De hecho son proporcionalmente más importantes en el centro metropolitano que en la periferia, lo que indica que a pesar de su rango y tamaño, Zaragoza sigue siendo un emplazamiento atractivo para empresas fabricantes de productos más estandarizados, sensibles a los costes salariales y al precio del suelo. Más de la mitad de tales empresas pertenecen a sectores tan tradicionales como el de alimentación y textil, que tienen en la ciudad el $74 \%$ y el $67 \%$, respectivamente, de los registros. En los demás sectores de bajo valor añadido el contraste es menos marcado y están en el centro metropolitano la mitad o menos de todas sus empresas. También tienen una alta implantación central algunas fabricaciones de valor añadido medio, como perfumes y productos de belleza e higiene o aparatos de sonido e imagen (con el 86\% y 79\%, respectivamente, de los registros correspondientes a ambas ramas).

El perfil productivo de la periferia metropolitana de Zaragoza es también más complejo que el descrito en los modelos teóricos. Junto a las escasas empresas de sectores de valor añadido alto, están más de la mitad de las de valor añadido medio, entre las que destacan las de caucho y materias plásticas, vehículos de motor y otro material de transporte, que sitúan en el entorno metropolitano el $67 \%, 74 \%$ y $67 \%$ por ciento de sus registros, respectivamente. Desde la implantación en 1981 de la multinacional General Motors, a 28 km al noroeste de Zaragoza, se ha generado un denso tejido de industrias proveedoras y auxiliares del automóvil, con municipios altamente especializados en ese tipo de actividades.

El resto de las empresas implantadas en el entorno metropolitano confirman lo previsto por la teoría ya que pertenecen a sectores de bajo valor añadido. Destacan las industrias de la madera, química y papel con más del $60 \%$ del total de sus respectivas implantaciones. En el caso de las industrias papeleras debe destacarse que no se trata en absoluto de empresas marginales, sino del complejo papelero más importante de España y uno de los más dinámicos de Europa. En éste y en otros casos las nuevas empresas se ven atraídas por la oferta municipal de suelo abundante, debidamente acondicionado y a precios competitivos, y por las subvenciones concedidas por el Gobierno de Aragón. 


\subsection{Influencia de la distancia en la desconcentración industrial metropolitana}

En el marco teórico presentado se establece que el área máxima de expansión o desconcentración industrial metropolitana se encuentra en un radio de 100 a $150 \mathrm{~km}$ o de una hora aproximadamente de distancia del centro metropolitano, dependiendo de las condiciones locales de las carreteras. En el caso de Zaragoza su alcance es sensiblemente menor. Como se explica seguidamente, el mayor dinamismo empresarial se ha localizado en municipios situados a menos de $30 \mathrm{~km}$ de Zaragoza. Ello se relaciona con aspectos ya señalados, como la extremada macrocefalia urbana (el hecho de que Zaragoza sea 45,7 veces más grande que el municipio que le sigue en población de su área metropolitana) y la extensión de su término municipal (que equivale al $31 \%$ de la superficie de la zona de estudio).

Para apreciar el efecto de la distancia sobre las implantaciones industriales se ha dividido el área metropolitana por coronas concéntricas y se ha calculado la proporción de empresas implantadas en los municipios situados dentro de cada corona (tabla 4). La primera constituye el centro de la aglomeración industrial y en ella se encuentra únicamente la ciudad de Zaragoza. Las demás son las coronas de expansión metropolitana. De los datos se deduce que el alejamiento del centro tiene un claro efecto disuasorio, ya que la proporción de registros disminuye con claridad desde el centro hacia la periferia del área metropolitana. Si se le da valor 100 a las implantaciones en la ciudad central, las de las sucesivas coronas tendrían, respectivamente, los valores 64, 22, 7 y 3.

Es en la segunda y tercera coronas donde tienen un mayor peso relativo los traslados de empresas. El hecho de que -como ya se ha comentadoprovengan del centro o de municipios de la primera corona (Escalona et al.,

Tabla 4: Distribución de las empresas según distancia a la ciudad central

\begin{tabular}{|c|c|c|r|r|r|c|}
\hline $\begin{array}{c}\text { DISTANCIA } \\
\text { (kilómetros) }\end{array}$ & $\begin{array}{c}\text { Distancia } \\
\text { (minutos) }\end{array}$ & $\begin{array}{c}\text { Nuevas } \\
\text { empresas }\end{array}$ & Traslados & $\begin{array}{c}\text { Total } \\
\text { empresas }\end{array}$ & $\%$ & $\begin{array}{c}\text { Relación } \\
\text { traslados/ } \\
\text { nuevas }\end{array}$ \\
\hline $0-5$ & $0-4$ & 1426 & 450 & 1876 & 49,7 & 0,32 \\
\hline $6-15$ & $5-12$ & 855 & 400 & 1255 & 33,3 & 0,47 \\
\hline $16-30$ & $13-25$ & 298 & 146 & 444 & 11,8 & 0,49 \\
\hline $31-45$ & $26-38$ & 114 & 23 & 137 & 3,6 & 0,20 \\
\hline $46-65$ & $39-55$ & 52 & 8 & 60 & 1,6 & 0,15 \\
\hline Total & & 2745 & 1027 & 3772 & 100,0 & 0,37 \\
\hline
\end{tabular}

Fuente: Registro de establecimientos industriales. 
2006) confirma la existencia de procesos centrífugos en la base de la desconcentración industrial metropolitana. No obstante, su alcance es limitado dado el descenso relativo de la importancia de los traslados en las coronas cuarta y quinta, descenso que no se compensa con las nuevas empresas, de modo que si no fuera por los municipios fuertemente industrializados de la primera corona (Cuarte y Cadrete, en el corredor sur; Utebo en el noroeste; La Puebla de Alfindén en el nordeste) o por los del corredor noroeste, donde los núcleos forman casi un continuum industrial, el alcance de la expansión industrial de Zaragoza sería aún más reducido.

No obstante, la atracción que mantiene la ciudad de Zaragoza sobre las industrias que se implantan en esta área no ha impedido su consolidación como una capital claramente terciaria, ni tampoco que en los municipios de su entorno se haya producido una marcada especialización industrial, resaltada por su tamaño incomparablemente menor, no sólo demográfico sino, sobre todo, funcional. Ello se refleja claramente en los cocientes de localización (figura 3), que se han calculado relacionando el número total de empresas industriales (las incluidas en el estudio y las existentes antes de 1992) con el número total de empresas (incluyendo todos los sectores económicos) existentes en la zona en el año 2006. Los valores obtenidos muestran que la mayor especialización industrial se da en el entorno inmediato de Zaragoza, observándose un gradiente significativo desde la segunda corona hasta las más periféricas. Desde este punto de vista el proceso de desconcentración industrial estaría más avanzado que lo que pudiera deducirse del manejo únicamente de los datos de distribución espacial por coronas.

La figura 3 muestra que la proporción de empresas industriales cae rápidamente desde la ciudad central hacia las coronas metropolitanas, concentrándose en aquélla las dos terceras partes del total y siendo casi irrelevante la proporción existente a partir de $30 \mathrm{~km}$. Pero, en cambio, la ciudad central muestra un menor nivel de especialización industrial, mientras que todas las coronas metropolitanas están claramente especializadas en industria, presentando el cociente de localización un perfil ascendente del centro a la segunda corona y luego descendente en función de la distancia, con valor máximo en la segunda $(2,75)$ y mínimo en la quinta $(1,02)$.

\section{Conclusión}

El análisis de las nuevas implantaciones industriales en el espacio metropolitano de Zaragoza, próximo a los 700000 habitantes, ha puesto de relieve que las grandes ciudades no son atractivas sólo ni fundamentalmente para las industrias de alto valor añadido que buscan economías de aglomeración, como 
Figura 3: Distribución espacial de la industria y cocientes de localización según la distancia en el entorno metropolitano de Zaragoza

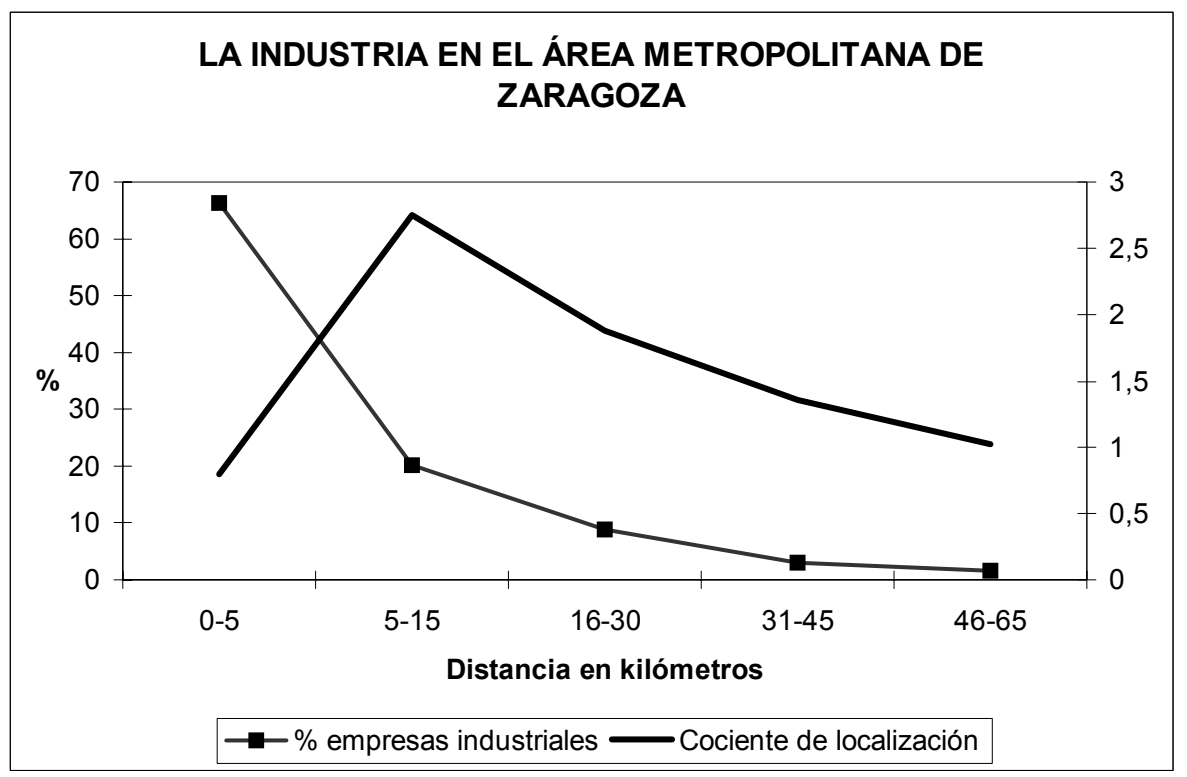

propone una de las hipótesis más extendidas en el momento actual. Por el contrario, el caso de Zaragoza muestra que las grandes metrópolis, incluso en sus áreas centrales, pueden ser también fuertemente atractivas para industrias de valor añadido medio y bajo, es decir, para industrias que buscan no tanto economías de aglomeración como bajos costes en mano de obra y suelo.

Se han señalado en el trabajo los factores que pueden explicar este doble atractivo de Zaragoza: por una parte, el tamaño de la ciudad (tanto demográfico como funcional, considerando en este caso especialmente la dotación de servicios) y las excelentes infraestructuras de transportes y comunicaciones aseguran a las empresas importantes economías de aglomeración. Por otra parte, la existencia de salarios bajos en relación con los de las principales aglomeraciones urbano-industriales españolas (Barcelona, Madrid y Bilbao) y su situación equidistante respecto a ellas la hacen atractiva para empresas que buscan bajos costes de la mano de obra. En tercer lugar, el disponer de un término municipal muy extenso le permite contar con una importante reserva de suelo que puede ser equipado para usos industriales y sacado al mercado a precios competitivos. Si a ello se añade, finalmente, la voluntad política de las autoridades locales, que en este caso han apostado por mantener la industria 
en la ciudad y no exclusivamente por su terciarización, el abanico de factores puede considerarse completo. Cabe esperar que si una ciudad grande cuenta con ventajas competitivas similares a las enumeradas sea atractiva para todo tipo de industrias, no sólo para las de alto valor añadido.

El análisis de Zaragoza demuestra también que una ciudad de tales características da lugar a procesos de desconcentración industrial de alcance espacial limitado, de manera que los nuevos establecimientos industriales de la aglomeración, tanto si se trata de empresas nuevas como de empresas trasladadas, siguen produciéndose mayoritariamente en la ciudad central y en las coronas periféricas inmediatas, sin que apenas rebasen los $30 \mathrm{~km}$, umbral de distancia muy por debajo de la isocrona de la hora que mencionan Polèse and Shearmour (2004) como umbral máximo de la desconcentración espacial. El nexo lógico entre ambos hechos es bastante claro: si en la ciudad central y su entorno inmediato resulta fácil encontrar suelo en condiciones económicas razonables y una mano de obra relativamente barata, las ventajas que en estos aspectos ofrecen las coronas exteriores del espacio metropolitano son insuficientes para compensar el incremento de los costes de interacción espacial que trae consigo su alejamiento del centro.

\section{Bibliografía}

AlCAlde InChausti, J. y P. AlCAlde Guindo (2007): Balance económico regional (autonomías y provincias) año 2000-2005, Fundación de las Cajas de Ahorro, Madrid.

Alonso-Logroño, M.P., E. Climent-López y A.I. Escalona-OrCaO (2006): «Zaragoza y el sector central del eje industrial del Ebro», en R. MÉNDEZ, y H. PASCUAL (coord.), Industria y ciudad en España. Nuevas realidades, nuevos retos, Thomson-Civitas, Madrid, pp. 557-586.

Ayuntamiento De Zaragoza (2002): Plan general de ordenación urbana de Zaragoza. Ayuntamiento de Zaragoza. Zaragoza.

CÁmARA de Comercio e Industria de Zaragoza (2006): [en línea] <http://www. camarazaragoza.com/poligonos/html/mapa_poligonos.asp>.

DiCKEN, P. (2004): Global shift, Sage, Londres.

Escalona-Orcao, A.I., E. Climent-López, B. Sánchez-Valverde, y P. MorenoMONTERDE (2006): «Perfiles empresariales y localización en el eje industrial del valle del Ebro», en Boletín de la Asociación de Geógrafos Españoles, n. ${ }^{\circ} 42$, pp. 31-52.

Escalona-Orcao, A.I., E. Climent-López, B. SÁnchez-Valverde y A. LlanosROBLES (2007): «Influencia de las políticas de suelo sobre la localización de industrias en áreas metropolitanas. El caso de Zaragoza», en Geocrítica. 
Scripta-Nova. Revista de Geografía y Ciencias Sociales, vol. XI, n. ${ }^{\circ}$ 240, [en línea] <http://www.ub.es/geocrit/sn/sn-240.htm>.

Graham, D. y N. SPENCE (1997): "Competition for metropolitan resources: the "crowding out" of London's manufacturing industry?», en Environment and Planning A, n. ${ }^{\circ} 29$, pp. 459-484.

Henderson, J.V. (1997): «Medium sized cities», en Regional Science and Urban Economics, n. ${ }^{\circ} 27$, pp. 583-612.

Holz, J.M. y J.P. Houssel (2003): Lindustrie dans la nouvelle économie mondiale. Presses Universitaires de France, París.

López, A., L.A. Castellano, C. DíEz y A. EsCalona (2002): «Carácter y localización de las nuevas empresas en el área metropolitana de Zaragoza: 1997$2000 »$, en Ería, n. ${ }^{\circ} 57$, p. 71-78.

MÉNDEZ GutiérRez DEL VALle, R. (2002): «Difusión de actividades y cambio metropolitano: el dinamismo de la franja periurbana en Madrid», en Boletín de la Real Sociedad Geográfica, n. ${ }^{\circ}$ 137-138, pp. 259-272.

MÉNDEZ, R. y S. SÁNCHEZ MORAL (2003): «Crisis, reconversión e integración en Europa, 1975-2000», en J. NADAL (dir.), Atlas de la industrialización de España, 1750-2000, Fundación BBVA-Crítica, Madrid.

MÉndeZ, R., J.C. García y J.J. Michelini (2005): «La nueva industria metropolitana. Tendencias y contrastes en la ciudad de Madrid», en Ería, n. ${ }^{\circ}$ 67, pp. 173-191.

NEL·LO, O. (1997): «Las grandes ciudades españolas: dinámicas urbanas e incidencia de las políticas estatales», en Papers. Regió Metropolitana de Barcelona, n. ${ }^{\circ} 27$, p. 9-70.

Pardos, E., F. Rubiera y A. GÓmEz (2004): «La localización de los servicios empresariales en Aragón. Determinantes y efectos», en Documento de Trabajo 5/2004, Fundación Economía Aragonesa, Zaragoza.

Polèse, M. and SHEARMOUR, R. (2004): «Is distance really dead? Comparing industrial location patterns over time in Canada», en International Regional Science Review, n. ${ }^{\circ} 27$ (4), pp. 431-457.

Polèse, M. y E. Champagne (1999): «Location matters. Comparing the distribution of economic activity in the Mexican and Canadian urban systems», en International Regional Science Review, n. ${ }^{\circ} 22$ (1), pp. 102-132.

POLĖSE, M., F. RUBIERA y R. SHEARMOUR (2005): Observing regularities in location patterns. An analysis of the spatial distribution of economic activity in Spain, Institut National de la Recherche Scientifique, Montreal, [en línea] <http:// www.inrs-ucs.uquebec.ca/pdf/inedit2005_08.pdf>.

Storper, M. y A. Venables (2002): «Buzz. The economic force of the city», en DRUID Summer conference: Industrial dynamics of the new and old economy. Who is embracing whom?, [en línea] <http://www.druid.dk/uploads/tx_picturedb/ ds2002-652.pdf>. 
VillaVERdE CASTRO, J. (2001): «El valle del Ebro como eje de desarrollo: una caracterización», en Papeles de Economía Española. Economía de las comunidades autónomas. El valle medio del Ebro, n. ${ }^{\circ} 19$, pp. 3-15. 